\title{
Effect of the COVID-19 Pandemic and Other
}

\section{Predictors of True Therapeutic Inertia on Patients} with Hypertension in a Primary Care Clinic in Thailand

\author{
Phoomjai Sornsenee (D) \\ Polathep Vichitkunakorn' \\ Kittisakdi Choomalee' \\ Chonticha Romyasamit ${ }^{2}$ \\ 'Department of Family and Preventive \\ Medicine, Faculty of Medicine, Prince of \\ Songkla University, Songkhla, Thailand; \\ ${ }^{2}$ Department of Medical Technology, \\ School of Allied Health Sciences, Walailak \\ University, Nakhon Si Thammarat, \\ Thailand
}

\begin{abstract}
Introduction: Hypertension (HT) has a significant impact on health care worldwide. Therapeutic inertia (TI) is defined as the failure to intensify therapy in the absence of an optimal goal and is widely used as a quality of care parameter. The coronavirus disease 2019 (COVID-19) pandemic has affected many health-care systems, including HT care. Therefore, the present study assessed the impact of the COVID-19 pandemic on TI and its predictors in patients with HT.

Methods: The electronic medical records of patients with HT who attended a primary care clinic at a tertiary hospital during pre-COVID-19 (February 2019 to February 2020) and COVID-19 (March to August 2020) periods were reviewed.

Results: Our study included 6089 visits during the 12-month pre-COVID-19 period and 2852 visits during the 6-month COVID-19 period. Most of the baseline characteristics of the HT patients were not significantly different between the two time periods. During the COVID-19 period, the percentage of uncontrolled HT visits decreased from $43 \%$ to $31 \%$. Similarly, the prevalence of TI decreased from $81 \%$ to $77 \%$. False TI was predominantly due to physicians' concerns regarding the in-clinic blood pressure measurement being inaccurate during both the periods.

Conclusion: After readjustment for the physicians 'reasons, the true TI was $64 \%$ and $60 \%$ in the pre-COVID-19 and COVID-19 period. For adjusted physician and patient-related factors, multilevel modeling was used. Senior medical staff visits, elderly patients, prior diabetes mellitus diagnosis, patients who used more than one type of anti-HT medication, and patients with systolic blood pressure $>150 \mathrm{mmHg}$ were all predictors of TI. The COVID19 period, on the other hand had no effect on TI with an adjusted odds ratio of 0.82 (95\% confidence interval, 0.67-1.01).
\end{abstract}

Keywords: hypertension, therapeutic inertia, COVID-19, quality of care, multilevel modeling, primary care

\section{Introduction}

Hypertension (HT) is a major noncommunicable disease (NCD) that causes significant mortality and morbidity worldwide. ${ }^{1,2}$ Less than half of all patients have controlled HT, despite being aware of the importance of optimal blood pressure (BP). ${ }^{3}$ As a result, the World Health Organization (WHO) has incorporated HT treatment quality into the Global Monitoring Framework for NCDs . ${ }^{4}$ A previous study highlighted three major factors that influence the quality of HT care: patients,
Correspondence: Phoomjai Sornsenee Tel +669542047/3

Email ezipnary@gmail.com 
clinicians, and the health-care system, which are all affected by various other factors. ${ }^{5}$

Therapeutic inertia (TI) is defined as failure of the health-care provider to intensify therapy in the absence of an optimal goal, such as inadequately adjusting antiHT medications to counteract increasing $\mathrm{BP}^{6,7}$ TI is widely used as a parameter to monitor the continued quality of HT care. ${ }^{8-10}$ Reducing TI in primary care might reduce its prevalence in populations with uncontrolled BP. ${ }^{11-13}$ The severity of HT, concern about adverse events, comorbidities, complexity of HT treatment guidelines, mean higher age of the patients, insurance, and treatment cost have been identified as predictors of TI. ${ }^{11,14}$

Patients' access to care has been affected by the coronavirus disease 2019 (COVID-19) pandemic due to the need for social distancing and lockdown measures. ${ }^{15,16} \mathrm{~A}$ rapid assessment survey from the WHO showed that $>50 \%$ of HT services were completely or partially disrupted due to the pandemic. ${ }^{17}$ Furthermore, $40 \%$ of NCD-related staff were deployed to provide COVID-19 relief. No previous studies have investigated whether the COVID-19 pandemic has influenced TI in HT care and whether TI predictors were the same during pre-COVID and COVID-19 periods. Understanding these issues is essential for healthcare planners to adjust the monitoring of quality of care in response to COVID-19.

The present study evaluated the effect of the COVID19 pandemic on TI among patients with HT in a primary care clinic (PCC) at a tertiary hospital in southern Thailand. The relationship between patient and physician-related factors on TI was also analyzed.

\section{Methods}

\section{Study Design and Setting, Participants, and the COVID-19 Situation}

We performed a retrospective cross-sectional study that included HT patients' visits to a PCC at the medical school at a tertiary hospital in Thailand. Most physicians in the PCC were family physicians, general practitioners, and internists working at the Department of Family and Preventive Medicine. All medical records in the hospital were fully computerized and retrievable through the Division of Digital Innovation and Data Analytics (DIDA) at the authors' institute.

COVID-19 cases were identified in Thailand at the beginning of 2019 and the disease spread widely from approximately March to April 2019. Therefore, the Thai government implemented measures to restrict interprovincial transportation and lock down high-risk areas. ${ }^{15}$ Postal prescriptions were provided to NCD patients undergoing continuous care who were unable to attend the hospital for any reason from April 2019 at the PCC.

\section{Data Collection}

We defined two study periods as the pre-COVID-19 period (February 2019 to February 2020) and COVID-19 period (March to August 2020). Data were anonymously extracted from the hospital database by DIDA. We identified HT visits from records with ICD-10 of Essential (primary) HT (I10) and/or use of anti-HT prescription. All medical records were reviewed.

The inclusion criteria were HT patients receiving continuous care at the PCC for more than two visits within the last six months. The exclusion criteria were records without BP records, patients whose relatives received medicine on their behalf, postal prescription, and nurse home visit prescription.

Data were under relational structure with each visit used as the main unit of analysis. Data regarding the patients' characteristics, such as gender, age, religion, insurance schemes, and comorbidities, were retrieved from the first registration visit. Data regarding the physicians' characteristics were extracted from the hospital database. On different occasions, each patient was seen by more than one physician and a physician usually attended to more than one patient.

\section{Operational Definitions of Key Variables}

1. Uncontrolled HT was defined as systolic blood pressure (SBP) $\geq 140 \mathrm{mmHg}$ and/or diastolic blood pressure (DBP) $\geq 90 \mathrm{mmHg}$ according to current guidelines. $^{18,19}$ Uncontrolled HT was identified by the selection of records in which these criteria were met.

2. TI was defined as uncontrolled HT in a patient who did not receive anti-HT treatment. This criterion did not apply to false TI in which the physician was fully aware of the uncontrolled HT yet did not add anti-HT medication for the following reasons (adapted from a previous study: ${ }^{7}$

(i) self-measured blood pressure monitoring (SMBP) at home data suggested that BP was well controlled at home;

(ii) concerns of in-clinic BP being inaccurate (data for repeat BP measurements in-clinic were shown in medical records);

(iii) patients refused to take additional medication; 
(iv) patients with a history of orthostatic hypotension; and

(v) anti-HT medication ran out (the patient had not been properly medicated before the visit).

The present study defined these patients as true TI if these reasons could not be found in the medical records.

The calculation of TI score was modified from the previous study. ${ }^{10}$ The patients who came to hospital more than two times in the 6 months of period were used in this study. Furthermore, true TI was used to calculate the TI score, which was defined as the adapted TI score. ${ }^{8,20}$

\section{Statistical Analysis}

Analyses were performed using $\mathrm{R}$ software version 4.0.2 with epiDisplay and lme4 packages. ${ }^{21,22}$ Baseline characteristics of the patients were compared across the two COVID-19 periods using $t$ test for continuous variables and chi-squared test for categorical variables to examine potential confounding roles of characteristics of the patients and the physician. The COVID-19 period was used as the stratification factor to examine the effects of other variables on TI, and predicting variables were compared between true TI and non-TI visits. The odds ratios (OR) from two strata were pooled together to calculate the period-adjusted OR of each variable using the MantelHaenszel method. Stratified linear regression was used for continuous data to estimate the pooled mean of that variable stratified by the COVID-19 period. Finally, multilevel logistic regression simultaneously considering the two factors was performed using the Linear MixedEffects Models in the lme4 package to adjust the effects of physician-related and patient-related variables. The final adjusted OR of COVID-19 period on TI, adjusted for both physician and patient-related variables and their $95 \%$ confidence interval $(95 \% \mathrm{CI})$ were reported. Statistical significance was considered when $P$-values were $<0.05$.

\section{Results}

There were 6089 visits during the 12-month pre-COVID19 period and 2852 during the 6-month COVID-19 period, and the average monthly visits were only reduced by $6 \%$ (Figure 1). However, during the 6-month COVID-19 period, $17.5 \%$ of the records indicated patients' absence at the clinics and $13 \%$ of visits included relatives who came to collect the medication prescribed physicians on behalf of the patients. Furthermore, $4 \%$ of patients had their medication delivered by post. These patients were not eligible for assessment of TI.

Table 1 shows a comparison of the baseline characteristics of HT patients who visited an attending physician at the PCC during the pre-COVID-19 and COVID-19 periods. There were no significant differences between the variables during the two periods except the percentage of thiazide prescribed and the percentage of prescriptions by

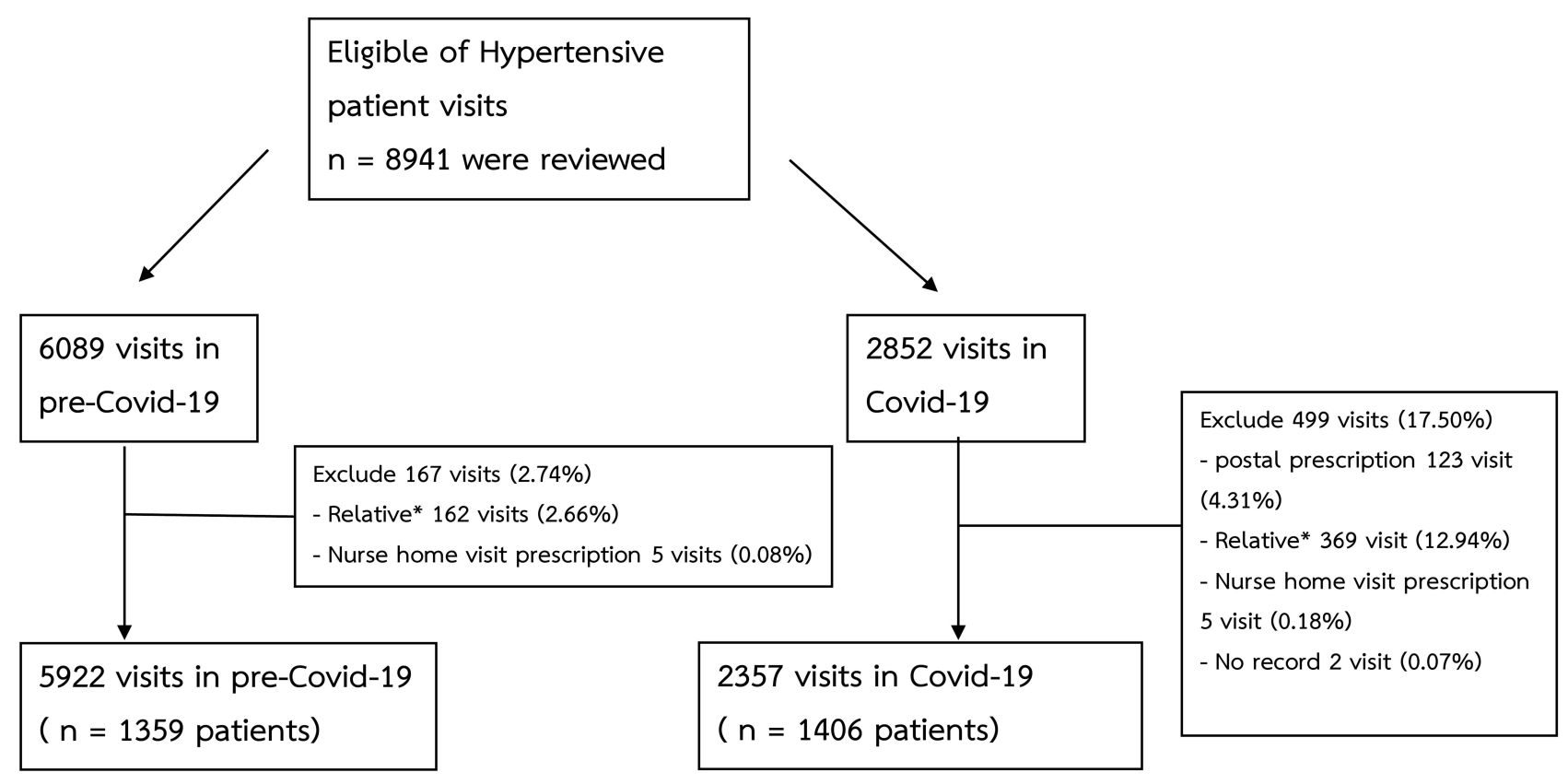

Figure I Patient enrollment between the pre-COVID-19 and COVID-19 periods. Note: $*$ Relative $=$ patients whose relatives received. 
Table I Baseline Characteristics of Patients with Hypertension and Physicians at the Primary Care Clinic During the Pre-COVID-19 and COVID-19 Periods

\begin{tabular}{|c|c|c|c|}
\hline Characteristics & Pre-COVID-19 (n = 1358) & COVID-19 $(n=1406)$ & $P$-value \\
\hline \multicolumn{4}{|l|}{ I.Characters of patients with $\mathrm{HT}$} \\
\hline Median age (IQR), years & $66.7(60.6,73.5)$ & $67(60.5,73.0)$ & 0.36 \\
\hline Male gender, no. (\%) & $505(37.2)$ & $524(37.3)$ & 0.996 \\
\hline Buddhist religion, no. (\%) & $1292(95.1)$ & $1340(95.3)$ & 0.979 \\
\hline \multicolumn{4}{|l|}{ Insurance scheme, no. (\%) } \\
\hline -Universal coverage & $523(38.6)$ & $589(41.9)$ & 0.425 \\
\hline -Civil servant medical benefit & $642(47.3)$ & $623(44.3)$ & \\
\hline -Social security & $53(3.9)$ & $60(4.3)$ & \\
\hline -Cash & $91(6.7)$ & $90(6.4)$ & \\
\hline -Other & $48(3.5)$ & $44(3.1)$ & \\
\hline Median body mass index (IQR) & $25.7(23.2,28.8)$ & $25.5(23.2,28.5)$ & 0.302 \\
\hline \multicolumn{4}{|l|}{ Comorbid, no. (\%) } \\
\hline -Diabetes mellitus & $539(39.7)$ & $540(38.4)$ & 0.514 \\
\hline -Dyslipidemia & | $247(9 \mid .8)$ & $1287(91.6)$ & 0.885 \\
\hline -Cardiovascular disease & $6(0.4)$ & $3(0.2)$ & $0.47 \mathrm{I}$ \\
\hline -Cerebrovascular disease & $39(2.9)$ & $34(2.4)$ & 0.532 \\
\hline -Chronic kidney disease stage $\geq 3$ & $280(26.3)$ & $280(25.5)$ & 0.9 \\
\hline \multicolumn{4}{|l|}{ Types of anti-HT medications, no. (\%) } \\
\hline -ACE-I & $449(33.1)$ & $424(30.2)$ & 0.109 \\
\hline$-A R B s$ & $440(32.4)$ & $500(35.6)$ & 0.087 \\
\hline$-\mathrm{CCBs}$ & $840(61.9)$ & $880(62.6)$ & 0.72 \\
\hline -Thiazides & $340(25.0)$ & $279(19.8)$ & $0.001 *$ \\
\hline -Alpha-blocker & $68(5.0)$ & $5 I(3.6)$ & 0.09 \\
\hline -Second-line drugs & $19(1.4)$ & $23(1.6)$ & 0.724 \\
\hline -Combine pill & $65(4.8)$ & $56(4.0)$ & 0.348 \\
\hline$\geq 2$ types of anti-HT medication & $688(50.7)$ & $667(47.4)$ & 0.098 \\
\hline \multicolumn{4}{|l|}{ 2.Characteristics of physicians } \\
\hline Median age (IQR), years & $30(28,34.5)$ & $29(28,32.5)$ & 0.538 \\
\hline Male gender, no. (\%) & $23(45.1)$ & $22(39.3)$ & 0.68 \\
\hline Median work experience (IQR), years & $6(4.0,11.0)$ & $5(4.0,8.2)$ & 0.557 \\
\hline Total of internist, no. (\%) & $31(60.8)$ & $37(66.1)$ & 0.714 \\
\hline Total of senior medical staff, no. (\%) & $14(27.5)$ & $13(23.2)$ & 0.779 \\
\hline \multicolumn{4}{|l|}{ Nature of visit } \\
\hline -Visit to internist, no. (\%) & $759(12.8)$ & $298(12.6)$ & 0.86 \\
\hline -Visit to senior medical staff, no. (\%) & $4624(78.1)$ & $1686(7 \mid .5)$ & $<0.00 I^{*}$ \\
\hline
\end{tabular}

Note: $* p<0.05$.

Abbreviations: ACE-I, angiotensin-converting enzyme inhibitors; ARBs, angiotensin receptor blockers; CCBs, calcium channel blockers; IQR, interquartile range.

senior staff (both were less common during the COVID-19 period).

Table 2 shows the BP parameters and TI during the pre-COVID-19 and pre-COVID-19 periods. The mean
SBP and DBP were significantly lower during the COVID-19 period. The percentage of uncontrolled HT visits decreased from $43 \%$ to $31 \%$ during the COVID-19 period. Similarly, the prevalence of TI decreased from 
Table 2 Blood Pressure Parameters and Therapeutic Inertia by COVID-19 Period (Visits)

\begin{tabular}{|c|c|c|c|c|}
\hline Parameters & Pre-COVID-I $9(n=5922)$ & COVID-19 $(n=2357)$ & Overall $(n=8279)$ & $P$-value* \\
\hline Median systolic blood pressure (IQR) & $137(128,146)$ & $133(125,|4|)$ & $136(127,145)$ & $<0.001$ \\
\hline Median diastolic blood pressure (IQR) & $75(68,82)$ & $73(66,8 I)$ & $75(67,82)$ & $<0.001$ \\
\hline No. of visits uncontrol hypertension, no. (\%) & $2569(43.4)$ & $739(31.4)$ & $3308(40)$ & $<0.001$ \\
\hline No. of TI visits, no. (\%) & $2080(81.0)$ & $565(76.5)$ & $2645(80)$ & 0.008 \\
\hline No. of true TI visits, no. (\%) & $1653(64.3)$ & $443(59.9)$ & $2096(63.3)$ & 0.033 \\
\hline Reason for false inertia & & & & 0.434 \\
\hline -SMBP at home & $63(14.8)$ & $24(19.7)$ & $87(15.8)$ & \\
\hline -Concerns of in-clinic BP being inaccurate & $290(67.9)$ & $73(59.8)$ & $363(66.1)$ & \\
\hline -Hypotension symptoms & $3(0.7)$ & $2(1.6)$ & $5(0.9)$ & \\
\hline -Refused medication & $5(1.2)$ & $\mathrm{I}(0.8)$ & $6(1.1)$ & \\
\hline -Ran out of medicine & $66(15.5)$ & $22(18)$ & $88(16)$ & \\
\hline Mean adapted TI score (SD) & $0.3(0.3)$ & $0.2(0.3)$ & $0.2(0.3)$ & $<0.001$ \\
\hline
\end{tabular}

Note: $* P<0.05$

Abbreviations: BP, blood pressure; IQR, interquartile range; SD, standard deviation; SMBP, self-measured blood pressure; TI, therapeutic inertia.

$81 \%$ to $77 \%$. False TI was predominantly due to physicians' concerns about the in-clinic BP being inaccurate during both periods and were not significantly different $(P$-value $=0.434)$. After readjustment for the physicians ${ }^{6}$ reasons, the true TI was $64 \%$ during the pre-COVID-19 period and $60 \%$ during the COVID- 19 period. The true TI and TI scores were significantly lower during the COVID19 period. For convenience, we used the true TI value as TI in subsequent analyses.

Table 3 illustrates the relationships between TI and patient and physician-related factors stratified by COVID-19 period. Since the interaction between the COVID-19 period and these variables was not significant (details of testing interaction effects are omitted), the pooled OR based on the Mantel-Haenszel method for categorical variables and the period-adjusted effect of continuous variables are displayed. TI visits were an average of $5.8 \mathrm{mmHg}(95 \% \mathrm{CI} 5.0-6.5)$ lower in SBP and $2.3 \mathrm{mmHg}(95 \%$ CI 3.0-5.0) lower in DBP compared with non-TI visits. Patients using ACE-inhibitors, second-line anti-HT medications, and those with a prior diagnosis of diabetes mellitus (DM) were associated with TI. TI visits were also attended by physicians with an average of 2.5 years more work experience and who were 2.5 years older than those attending non-TI visits. Senior medical staff ( $>10$ years' experience) showed an increased risk of true TI. On the other hand, internists were associated with a protective effect, with an adjusted OR of 0.5 (95\% CI $0.32-0.85)$.
Table 4 shows the final model predicting TI using multilevel (lme4) modeling after simultaneously and mutually adjusting for physician and patient-related factors. Five significant predictors for TI included senior medical staff visits, elderly patients, patients with prior DM diagnosis, patients who used more than one type of anti-HT medication, and patients with SBP $>150 \mathrm{mmHg}$.

The COVID-19 period had an adjusted OR of 0.82 (95\% CI 0.67-1.01). Thus, there was insufficient evidence to state whether the COVID-19 period was associated with an increase in TI.

\section{Discussion}

\section{Statement of Principal Findings}

In our clinical setting, the COVID-19 pandemic resulted in nearly one-sixth of prescriptions being made without the physician really seeing the patients. The demographic backgrounds of the patients and the physicians during the COVID-19 period were not significantly different from those in the preceding period. On the other hand, patients visiting the PCC during the COVID-19 period had lower SBP, DBP, uncontrolled HT and greater use of thiazides. Most cases of false TI were due to physicians' concerns about the inaccuracy of the in-clinic BP during both periods. During both periods, TI was consistently more common in elderly patients and those with a prior DM diagnosis. Patients with SBP $>150 \mathrm{mmHg}$ were three times less likely to experience TI compared with those 


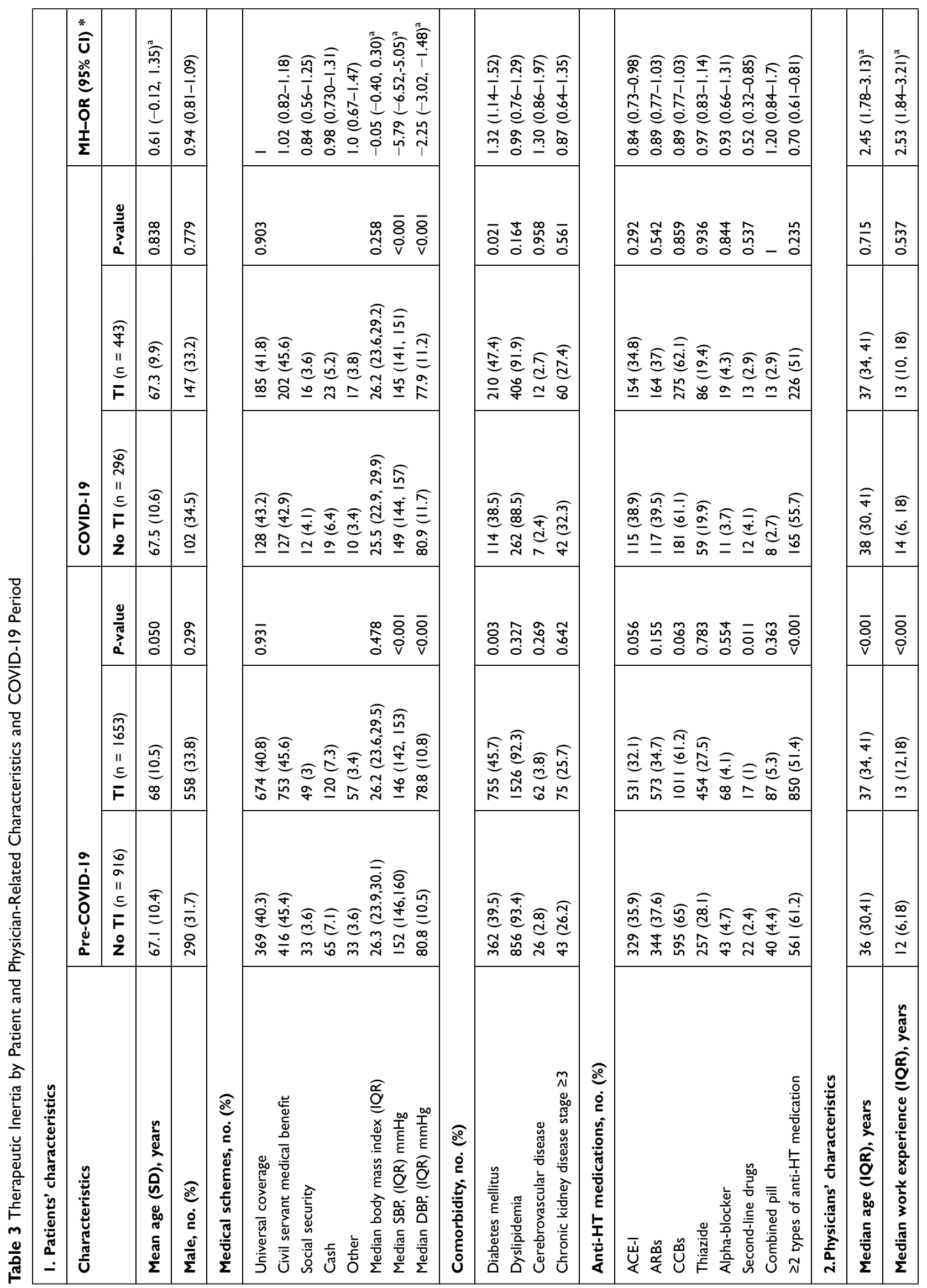




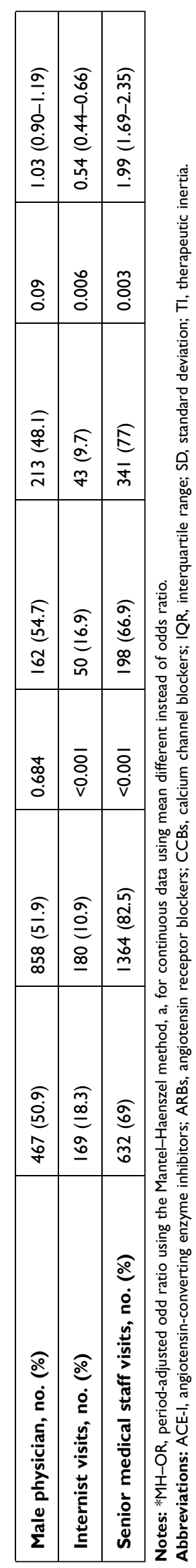

with SBP of 141-150 mmHg. The adjusted OR of TI also increased two-fold among patients attended by senior staff. Patients who used more than one type of anti-HT therapy were less likely to experience TI compared with those using only one type. Finally, there was no relationship between COVID-19 period and TI after adjusting for factors using the multilevel model.

\section{Interpretation Within the Context of the Wider Literature}

The effect of COVID-19 may not contribute to demographic factors. However, our study found that BP parameters, percentage of uncontrolled HT visits, and TI of patients during the COVID-19 period were significantly better than during the pre-COVID-19 period. Patients who are socioeconomically vulnerable are more likely to become non-adherent in the absence of effective public health interventions. ${ }^{23,24}$ In the present study, the government measures during the COVID-19 period included only those patients who were able to come to the hospital. Therefore, vulnerable patients who were unable to access health care during the pandemic may have been excluded from the study. The main cause of false TI was physicians' concerns about the BP measured in the clinic being inaccurate, which is consistent with the reports of previous studies. ${ }^{8,9}$ HT treatment should be designed as a framework for physicians to improve the outcome of HT care and reduce TI. ${ }^{9}$ However, appropriated TI should be more accurate if patients are encouraged to use SMBP at home, as the present study revealed that this is the second greatest cause of false TI. Furthermore, current clinical guidelines indicate that SMBP is effective in lowering BP. ${ }^{25}$

The effect of the COVID-19 period on TI was not found to be significant after adjustment for patient and physician-related factors. On the other hand, the findings of our study were consistent with those of previous studies, which stated that TI could be influenced by the patient-associated factors, such as comorbidities, severity of HT, and age. ${ }^{14}$ Novel factors, such as senior medical staff and one type of anti-HT medication usage, were found to strongly affect TI. There have been no direct studies on the association between senior staff and TI; however, it is possible that senior medical staff are concerned about the impact of long-term doctor-patient relationships and acknowledge patient preferences, resulting in a slower rate of medication increase. ${ }^{26}$ 
Table 4 Factors Associated with Therapeutic Inertia in Hypertension Treatment Using Final Multilevel Modeling

\begin{tabular}{|c|c|}
\hline Factor & $\begin{array}{l}\text { Adjusted OR } \\
(95 \% \mathrm{Cl})\end{array}$ \\
\hline Intercept & $1.05(0.40-2.79)$ \\
\hline \multicolumn{2}{|l|}{ I. Patient-related } \\
\hline COVID-19 vs pre-COVID-19 period & $0.82(0.67-1.01)$ \\
\hline Female vs male & $0.98(0.81-1.18)$ \\
\hline Increase in I year of age & $1.01(1.00-1.02)$ \\
\hline SBP >I50 mmHg vs SBP $14 \mid-150 \mathrm{mmHg}$ & $0.34(0.28-0.4 \mathrm{I})$ \\
\hline DM vs non-DM & $1.43(1.19-1.72)$ \\
\hline Using $\geq 2$ vs I type of $\mathrm{HT}$ medication & $0.65(0.54-0.78)$ \\
\hline $\begin{array}{l}\text { Using second-line vs other anti-HT } \\
\text { medication }\end{array}$ & $0.56(0.31-1.03)$ \\
\hline Using ACE-I vs other anti-HT medication & I.0I (0.84-I.22) \\
\hline \multicolumn{2}{|l|}{ 2. Physician-related } \\
\hline Senior staff vs other physicians & $1.87(1.06-3.31)$ \\
\hline Female vs male & $0.96(0.65-1.42)$ \\
\hline Internist vs other physician & $0.83(0.45-1.52)$ \\
\hline
\end{tabular}

Abbreviations: ACE-I, angiotensin-converting enzyme inhibitors; $\mathrm{Cl}$, confidence interval; DM, diabetes mellitus; HT, hypertension, OR, odds ratio; SBP, systemic blood pressure.

\section{Strengths and Limitations}

One limitation of this study is that it was a retrospective cross-sectional study in which data were reviewed from medical records and the reasons that physicians prescribed anti-HT were assumed from their notes. However, in clinical practice, physicians did not always record the reasons. Therefore, this retrospective study was limited by incomplete information in the medical records.

A strength of the present study is that it is the first to identify a relationship between the COVID-19 pandemic and TI in HT care. However, COVID-19 outcomes are not directly relevant to TI. Nevertheless, we revealed intriguing effects of COVID-19 that contribute to HT care, such as an increased percentage of uncontrolled BP and other predictors for TI.

\section{Implications for Policy, Practice, and Research}

Our findings revealed that physicians were concerned about increasing anti-HT in patients with SBP $>150 \mathrm{mmHg}$, even though the current HT guidelines recommend a lower optimal BP. ${ }^{18,19}$ This encourages physicians to follow current HT guidelines more rigorously. The guidelines demonstrate the clear benefits of combining anti-HT types for BP control, particularly in uncontrolled HT. However, in the present study, TI was higher with use of one type of anti-HT medication compared with use of two or more types. This demonstrates the reluctance of physicians to increase anti-HT use from one to two types. On the other hand, the prompt addition of a second type of anti-HT medication makes the next step simpler. Our findings showed a positive effect of senior staff on increasing TI. A previous study suggested that one of main problems was providers' own clinical judgment and experience. ${ }^{26}$ However, there has been no prior research directly describing TI among senior staffs. We hypothesized that senior staff may have had a long relationship with their patients, due to which rapid medication adjustment would not have been performed but they might suggest the patient to lifestyle modifications.

To resolve this issue, we propose that the implementation and revision of a protocol for improved HT control in primary care in the HT clinic as part of routine evidence-based practice. Further prospective studies are required using TI or TI score to represent health-care system inertia in other NCDs, including HT. In addition, we propose that future studies should include an intervention to improve TI in NCD care. Finally, in our study setting, we demonstrated that resilience and adapted health care was strongly exhibited during the pandemic. This developing systematic approaches are in accordance with the digital healthcare solution for NCD services during the pandemic of WHO. ${ }^{17}$

However, while out-of-patient prescriptions may have a negative impact on the quality of HT care, it may benefit coverage during a pandemic. Finding a balance between treatment quality and access to treatment should be considered concurrently for COVID-19 as well as the next pandemic. The health-care system should be well-prepared and efficient.

\section{Conclusion}

The present study revealed that the COVID-19 pandemic had no significant effect on TI in HT care. Senior staff were positive for TI among the physician-related factors. Furthermore, patient-related factors, such as elderly patients, prior DM diagnosis, lower SBP, and use of only one medication were associated with TI. However, the medical health care in this study was adapted to deal with the pandemic. 


\section{Key Messages}

- The coronavirus disease-2019 (COVID-19) pandemic has affected many health-care systems, including hypertension care (HT). Therapeutic inertia (TI) is defined as failure to intensify therapy in the absence of an optimal goal. This study assessed the impact of the pandemic on TI and its predictors in patients with HT in a primary care clinic (PCC) in Thailand.

- The study found that the demographics of patients and physicians during the COVID-19 period were not significantly different from those in the preceding period. During the COVID-19 period, patients visiting the PCC had lower blood pressure and uncontrolled HT.

- TI was consistently more common in elderly patients and those with a prior diabetes mellitus diagnosis during both the periods. The adjusted odds ratio of TI increased by a factor of two among patients treated by senior staff. Patients who received more than one type of anti-HT therapy were less likely to develop TI than those who received only one type.

- However, after adjusting for factors using the multilevel model, no relationship was found between the COVID-19 period and TI.

\section{Data Sharing Statement}

The data presented in this study are available from the corresponding authors upon reasonable request.

\section{Ethics and Other Permissions}

The study was approved by the Human Research ethics committee (HREC), Faculty of Medicine, Prince of Songkla University issued on October 20, 2020. (Approval Number: REC 63-396-9-1). HREC is an official institute responsible for ethical review and supervision for human research. The study was conducted in accordance with the Declaration of Helsinki. All medical records were fully computerized by the Division of Digital Innovation and Data Analytics and confidentially reported.

\section{Informed Consent Statement}

The requirement for informed consent was waived because of the retrospective study design. This retrospective study was conducted using electronic medical records. The clinical data that could be used to identify patients was kept private, and the record form was assigned a code number. The patients' code numbers were used during the analysis.

\section{Acknowledgments}

We would like to express our gratitude to the Faculty of Medicine at Prince of Songkla University for encouraging, practicing, and assisting young medical staff in conducting research. We would also like to thank Professor Virasakdi Chongsuvivatwong and Assistant Professor Paramee Thongsuksai who provided us with useful advice for conducting this research.

\section{Author Contributions}

All authors made a significant contribution to the work reported, whether that is in the conception, study design, execution, acquisition of data, analysis and interpretation, or in all these areas; took part in drafting, revising or critically reviewing the article; gave final approval of the version to be published; have agreed on the journal to which the article has been submitted; and agree to be accountable for all aspects of the work.

\section{Funding}

This research was financially supported by the Faculty of Medicine, Prince of Songkla University, Thailand.

\section{Disclosure}

The authors report no conflicts of interest in this work.

\section{References}

1. Benjamin EJ, Muntner P, Alonso A, et al. Heart disease and stroke statistics2019 update: a report from the American heart association. Circulation. 2019;139(10):e56-e528. doi:10.1161/CIR.0000000000000659

2. Bromfield S, Muntner P. High blood pressure: the leading global burden of disease risk factor and the need for worldwide prevention programs. Curr Hypertens Rep. 2013;15(3):134-136. doi:10.1007/ s11906-013-0340-9

3. Fryar CD, Ostchega Y, Hales CM, Zhang G, Kruszon-Moran D. Hypertension Prevalence and control among adults: United States, 2015-2016. NCHS Data Brief. 2017;289:1-8.

4. World Health Organization. Global status report on noncommunicable diseases 2014. Availablefrom: https:/www.who.int/nmh/publications/ ncd-status-report-2014/en/. Accessed August 31, 2021.

5. Milani RV, Lavie CJ, Wilt JK, Bober RM, Ventura HO. New concepts in hypertension management: a population-based perspective. Prog Cardiovasc Dis. 2016;59(3):289-294. doi:10.1016/j. pcad.2016.09.005

6. Lebeau J-P, Cadwallader J-S, Aubin-Auger I, et al. The concept and definition of therapeutic inertia in hypertension in primary care: a qualitative systematic review. BMC Fam Pract. 2014;15:130. doi:10.1186/1471-2296-15-130

7. Phillips LS, Branch WT, Cook CB, et al. Clinical inertia. Ann Intern Med. 2001;135(9):825-834. doi:10.7326/0003-4819-135-9-200111060-00012

8. Desai N, Madhavankutty Saraswathy V, Hunter K, McFadden C. Prevalence of true therapeutic inertia in blood pressure control in an academic chronic kidney disease clinic. J Clin Hypertension. 2013a;15 (6):375-379. doi:10.1111/jch.12095 
9. Egan BM, Sutherland SE, Rakotz M, et al. Improving hypertension control in primary care with the measure accurately, act rapidly, and partner with patients protocol. Hypertension. 2018;72(6):1320-1327. doi:10.1161/HYPERTENSIONAHA.118.11558

10. Okonofua EC, Simpson KN, Jesri A, Rehman SU, Durkalski VL, Egan BM. Therapeutic inertia is an impediment to achieving the Healthy People 2010 blood pressure control goals. Hypertension. 2006a;47(3):345-351. doi:10.1161/01.HYP.0000200702.76436.4b

11. Boonyasai RT, Rakotz MK, Lubomski LH, et al. Measure accurately, Act rapidly, and Partner with patients: an intuitive and practical threepart framework to guide efforts to improve hypertension control. $J$ Clin Hypertension. 2017;19(7):684-694. doi:10.1111/jch.12995

12. Milman T, Joundi RA, Alotaibi NM, Saposnik G. Clinical inertia in the pharmacological management of hypertension: a systematic review and meta-analysis. Medicine. 2018;97(25):e11121. doi:10.1097/MD.0000000000011121

13. Wang YR, Alexander GC, Stafford RS. Outpatient hypertension treatment, treatment intensification, and control in Western Europe and the United States. Arch Intern Med. 2007;167(2):141-147. doi:10.1001/archinte.167.2.141

14. Feldman RD, Liu L, Wu Z, Zhang Y, Yu X, Zhang X-H. Hypertension Attitude PersPEctives and Needs (HAPPEN): a Real-World Survey of Physicians and Patients With Hypertension in China. J Clin Hypertension. 2017;19(3):256-264. doi:10.1111/jch.12912

15. Dechsupa S, Assawakosri S, Phakham S, Honsawek S. Positive impact of lockdown on COVID-19 outbreak in Thailand. Travel Med Infect Dis. 2020;36:101802. doi:10.1016/j.tmaid.2020.101802

16. Modesti PA, Wang J, Damasceno A, et al. Indirect implications of COVID-19 prevention strategies on non-communicable diseases: an Opinion Paper of the European Society of Hypertension Working Group on Hypertension and Cardiovascular Risk Assessment in Subjects Living in or Emigrating from Low Resource Settings. BMC Med. 2020;18(1):256. doi:10.1186/s12916-020-01723-6

17. World Health Organization. The impact of the COVID-19 pandemic on noncommunicable disease resources and services: results of a rapid assessment; 2020. Availablefrom: https://www.who.int/publica tions/i/item/9789240010291. Accessed August 31, 2021.
18. Thai Hypertension Society. 2019 Thai Guidelines on The Treatment of Hypertension; 2019. Availablefrom: http://www.thaihypertension. org/files/HT\%20guideline\%202019.with\%20watermark.pdf. Accessed August 31, 2021.

19. Unger T, Borghi C, Charchar F, et al. 2020 International Society of Hypertension global hypertension practice guidelines. Hypertension. 2020;75(6):1334-1357. doi:10.1161/HYPERTENSIONAHA.120.15026

20. Okonofua EC, Simpson KN, Jesri A, Rehman SU, Durkalski VL, Egan BM. Therapeutic Inertia is an impediment to achieving the healthy people 2010 blood pressure control goals. Hypertension. 2006b;47(3):345-351. doi:10.1161/01.HYP.0000200702.76436.4b

21. Bates D, Sarkar D, Bates MD, Matrix L. The lme4 package. $R$ Package Version. 2007;2(1):74.

22. Chongsuvivatwong V. EpiDisplay: Epidemiological Data Display Package (R Package Version 3.5. 0.1.). 2018.

23. Basu S. Non-communicable disease management in vulnerable patients during Covid-19. Indian J Med Ethics. 2020;5(2):103-105. doi:10.20529/IJME.2020.041

24. Chan EYY, Kim JH, Lo ESK, et al. What Happened to People with Non-Communicable Diseases during COVID-19: implications of H-EDRM Policies. Int $J$ Environ Res Public Health. 2020;17:15. doi:10.3390/ijerph17155588

25. Daichi S, Artinian Nancy T, Basile Jan N, et al. Self-Measured blood pressure monitoring at home: a joint policy statement from the American heart association and American medical association. Circulation. 2020;142(4):e42-e63. doi:10.1161/CIR.0000000000000803

26. Aujoulat I, Jacquemin P, Rietzschel E, et al. Factors associated with clinical inertia: an integrative review. Adv Med Educ Practice. 2014;5:141. doi:10.2147/AMEP.S59022
Risk Management and Healthcare Policy

\section{Publish your work in this journal}

Risk Management and Healthcare Policy is an international, peerreviewed, open access journal focusing on all aspects of public health, policy, and preventative measures to promote good health and improve morbidity and mortality in the population. The journal welcomes submitted papers covering original research, basic science, clinical \& epidemiological studies, reviews and evaluations,

\section{Dovepress}

guidelines, expert opinion and commentary, case reports and extended reports. The manuscript management system is completely online and includes a very quick and fair peer-review system, which is all easy to use. Visit http://www.dovepress.com/testimonials.php to read real quotes from published authors. 